Proceedings of the 46th International School and Conference on the Physics of Semiconductors "Jaszowiec" 2017, Szczyrk

\title{
Magnetic and Structural Studies of GeMnSnTe Epitaxial Layers
}

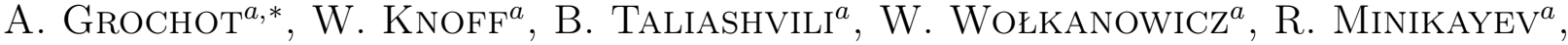

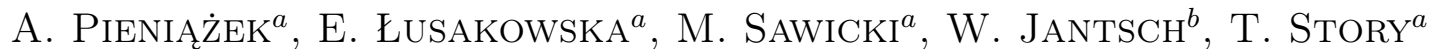 \\ AND H. PRZYBYLIŃSKA ${ }^{a}$ \\ ${ }^{a}$ Institute of Physics, Polish Academy of Sciences, aleja Lotnikow 32/46, PL-02668 Warsaw, Poland \\ ${ }^{b}$ Institute of Semiconductor and Solid State Physics, Johannes Kepler University, Linz, Austria \\ MBE grown $\mathrm{Ge}_{1-x-y} \mathrm{Mn}_{x} \mathrm{Sn}_{y}$ Te layers with Mn content ranging from 10 to $30 \%$ and Sn content ranging \\ from 2 to $5 \%$ have been characterized with X-ray diffraction, energy-dispersive X-Ray spectroscopy, atomic force \\ microscopy, SQUID magnetometry, and ferromagnetic resonance. All layers (except the one with the highest Mn \\ and Sn content) were found to be single phase rhombohedral, with the distortion axis perpendicular to the layer \\ surface, and ferromagnetic. Ferromagnetic resonance studies have shown that co-doping with a few percent of tin \\ makes the lattice more rigid and changes considerably the magnetocrystalline anisotropy, from purely uniaxial in \\ GeMnTe to distorted cubic in $\mathrm{Ge}_{1-x-y} \mathrm{Mn}_{x} \mathrm{Sn}_{y} \mathrm{Te}$ at the same Mn content.
}

DOI: 10.12693/APhysPolA.132.340

PACS/topics: 75.30.Gw, 75.50.Pp, 76.50.+g

\section{Introduction}

Multiferroics - materials in which ferromagnetism and ferroelectricity coexist $[1-3]$ - provide extraordinary physical properties due to the mutual coupling between ferromagnetic (FM) and ferroelectric (FE) degrees of freedom. This gives rise to new magnetoelectric effects that are not only of great fundamental interest but also provide many potentials for device applications [4-6], including programmable logic, nonvolatile memories, as well as spintronic devices based on the Rashba and Zeeman effects. Single phase multiferroics exhibiting simultaneous ferroelectricity and ferromagnetism are, however, very rare due to a fundamental contradiction: a cation in a crystal lattice can move off-center to induce ferroelectricity as long as it has an empty $d$ shell [7] whereas ferromagnetism in transition metal compounds requires ions with partly filled $d$ shells. This apparent contradiction is lifted in magnetically doped $\mathrm{Ge}_{1-x} \mathrm{Mn}_{x}$ Te. In this ternary alloy, the $\mathrm{Ge}^{2+}$ ions $\left(3 d^{10}\right)$ induce the ferroelectricity and the $\mathrm{Mn}^{2+}$ ions $\left(3 d^{5}\right)$ the ferromagnetism, providing the magnetic moments that couple to each other via the free carrier mediated Ruderman-Kittel-KasuyaYosida (RKKY) interaction [8]. As recently demonstrated with the use of ferromagnetic resonance [9], the FE and FM moments interact leading to abrupt switching of ferroelectric domain orientation under applied magnetic field. In order to gain more insight into the mechanism driving, the domain switching quaternary GeMnSnTe layers were grown, with the aim to study domain switching close to the ferroelectric (rhombohedral) to paraelectric (cubic) phase transition. This is not possible

*corresponding author; e-mail: grochot@ifpan.edu.pl in GeMnTe, since the ferroelectric Curie temperature, $T_{\mathrm{C}}^{f e}$, exceeds the ferromagnetic one, $T_{\mathrm{C}}^{f m}$. In SnTe $T_{\mathrm{C}}^{f e}$ is about $145 \mathrm{~K} \mathrm{[10],} \mathrm{which} \mathrm{is} \mathrm{considerably} \mathrm{lower} \mathrm{than}$ in GeTe. SnMnTe is ferromagnetic with $T_{\mathrm{C}}^{f m}$ less than $7 \mathrm{~K}$ [11]. Quaternary GeMnSnTe alloys grown by a modified Bridgman technique have been studied before and found to exhibit spin-glass like behavior at low temperatures [12]. Here we report preliminary results obtained for ferromagnetic and ferroelectric GeMnSnTe layers grown by molecular beam epitaxy (MBE).

\section{Experimental}

The $1 \mu \mathrm{m}$ thick $\mathrm{Ge}_{1-x-y} \mathrm{Mn}_{x} \mathrm{Sn}_{y}$ Te layers were grown on (111) $\mathrm{BaF}_{2}$ substrates, with Mn content $x$ ranging from 10 to $30 \%$ and Sn content $y$ ranging from 2 to $5 \%$. X-ray diffraction and atomic force microscopy investigations have proven excellent crystalline quality of the layers. The chemical composition was determined with energy-dispersive X-Ray spectroscopy (EDX) and found to be uniform across the layers. However, the Xray diffraction (XRD) peaks shown in Fig. 1 are evidently broadened and even visibly split, indicating some inhomogeneity in the dopant distribution. Nevertheless, the positions of the maxima agree well with those detected in Sn-free GeMnTe layers [9] for the same manganese concentration as indicated by EDX, except for the layer with the highest Mn content.

\section{Results}

All the grown layers were found to be ferromagnetic at low temperatures, both by ferromagnetic resonance (FMR) experiments as well as by SQUID magnetometry. In FMR, however, weak signals stemming from regions with evidently lower Mn concentration than the dominant one were also detected. The additional signals disappeared at about $10 \mathrm{~K}$, due to the lower ferromagnetic 




Fig. 1. X-ray diffraction patterns $(\omega-2 \theta$ scans $)$ of $\mathrm{Ge}_{1-x-y} \mathrm{Mn}_{x} \mathrm{Sn}_{y}$ Te layers at room temperature. Sharp peaks are from the $\mathrm{BaF}_{2}$ substrate. The $\mathrm{Mn}$ and $\mathrm{Sn}$ contents determined with EDX are given in the figure. The spectra are shifted vertically for clarity.

Curie temperature. The temperature dependence of inplane magnetization measured for $\mathrm{Ge}_{0.88} \mathrm{Mn}_{0.1} \mathrm{Sn}_{0.02} \mathrm{Te}$ and $\mathrm{Ge}_{0.65} \mathrm{Mn}_{0.3} \mathrm{Sn}_{0.05}$ Te layers is shown in Fig. 2a. Since the easy magnetization axis for the layers up to $\mathrm{Mn}$ content of $x=0.27$ is out-of-plane, the measurements were performed at an applied magnetic field of $1 \mathrm{kOe}$ to ensure in-plane orientation of the magnetic moment. The in-plane hysteresis loops for the same samples measured at $3 \mathrm{~K}$ are presented in Fig. 2b. Both the $M(T)$ and $M(H)$ behavior of the $\mathrm{Ge}_{0.79} \mathrm{Mn}_{0.17} \mathrm{Sn}_{0.04} \mathrm{Te}$ and $\mathrm{Ge}_{0.695} \mathrm{Mn}_{0.27} \mathrm{Sn}_{0.035}$ Te layers is similar to that of $\mathrm{Ge}_{0.88} \mathrm{Mn}_{0.1} \mathrm{Sn}_{0.02} \mathrm{Te}$. In contrast, the magnetic moment in the $\mathrm{Ge}_{0.65} \mathrm{Mn}_{0.3} \mathrm{Sn}_{0.05}$ Te layer is oriented in-plane even without an externally applied magnetic field and the $M(H)$ dependence exhibits no hysteresis. Moreover, ferromagnetic resonance in this layer is observed only for magnetic field directions close to in-plane. Such a behavior is typical for an uncompensated antiferromagnet and together with the high Curie temperature (see Fig. 2b) suggests precipitatation of zinc-blende MnTe phase.

More information on magnetic anisotropy of the GeMnSnTe layers was obtained from angular dependent ferromagnetic resonance studies. In the experiment the microwave magnetic field $\boldsymbol{H}_{m w}$ was oriented in-plane al-

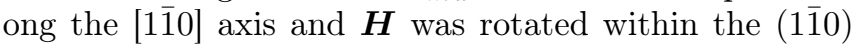
plane. This plane contains four important crystallographic axes: the [111] normal and the oblique [11]], [110], [001] directions. The angular dependences of the FMR peak positions for the two layers with the most uniform $\mathrm{Mn}$ distribution, i.e., $\mathrm{Ge}_{0.88} \mathrm{Mn}_{0.1} \mathrm{Sn}_{0.02} \mathrm{Te}$ and $\mathrm{Ge}_{0.79} \mathrm{Mn}_{0.17} \mathrm{Sn}_{0.04} \mathrm{Te}$, are shown in Figs. 3 and 4 , respectively. The solid lines are fits to the experimental points with the following formula determining the resonance condition:

$$
h \nu=\left.\frac{g \mu_{\mathrm{B}}}{M \sin \theta_{e q}} \sqrt{F_{\theta \theta} F_{\phi \phi}-F_{\theta \phi}^{2}}\right|_{\theta_{e q}, \phi_{e q}},
$$

where $\nu$ is the resonance frequency, $h$ - the Planck constant, $\mu_{\mathrm{B}}$ - the Bohr magneton, $g$ is the spectroscopic
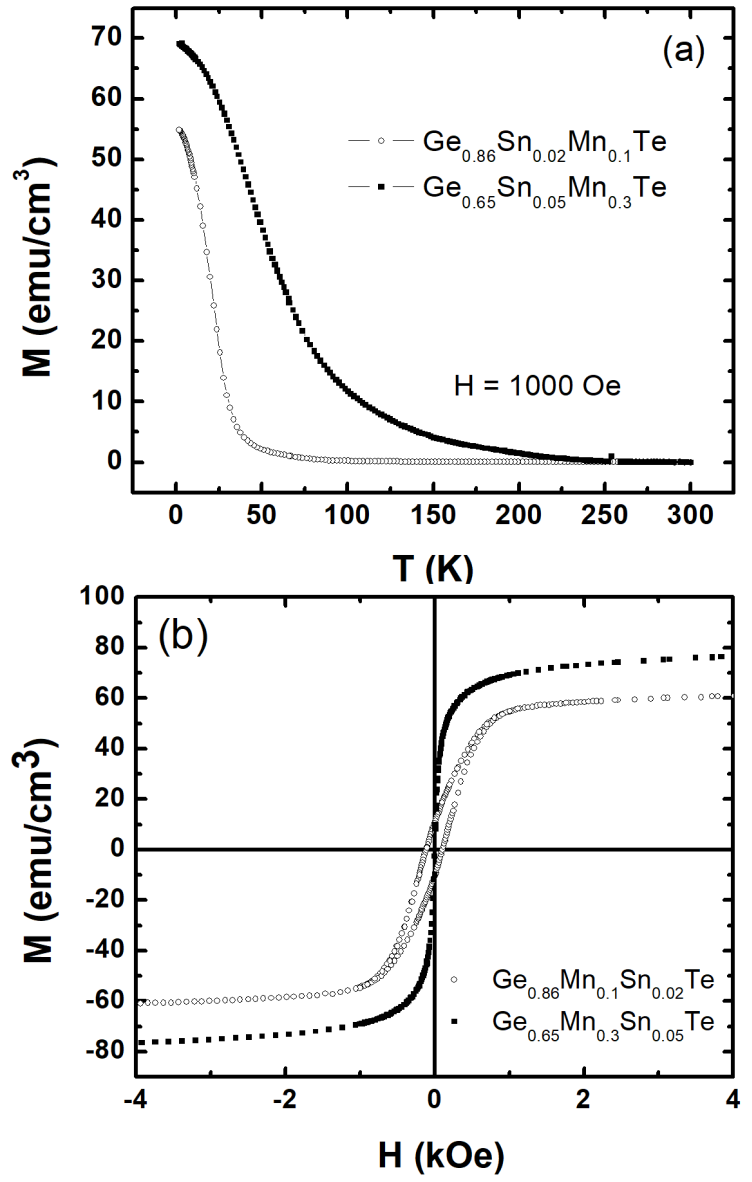

Fig. 2. Temperature dependent magnetization at $1 \mathrm{kOe}(\mathrm{a})$ and hysteresis loops at $3 \mathrm{~K}$ (b) for two GeMnSnTe layers with the lowest and the highest Mn content. The magnetic field was applied in-plane.

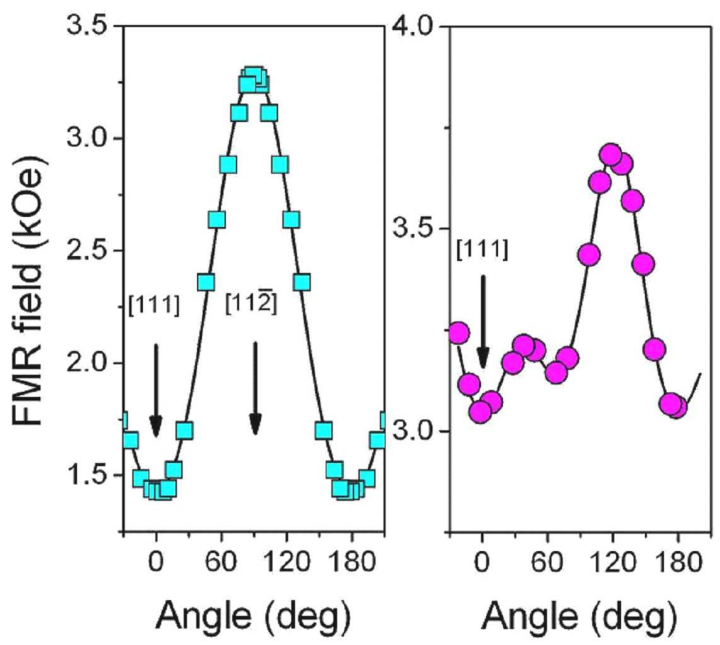

Fig. 3. FMR peak positions in $\mathrm{Ge}_{0.85} \mathrm{Mn}_{0.15} \mathrm{Te}$ (left) and $\mathrm{Ge}_{0.86} \mathrm{Mn}_{0.1} \mathrm{Sn}_{0.04} \mathrm{Te}$ (right) layers vs. orientation of the applied magnetic field, $\mathbf{H}$, in the (1110) plane at 3 and $10 \mathrm{~K}$, respectively. The angle is measured from the [111] direction (c-axis) perpendicular to the layer surface. The solid lines are fits assuming MAE of 2nd order for $\mathrm{Ge}_{0.85} \mathrm{Mn}_{0.15} \mathrm{Te}$ and 2nd and 4th order for $\mathrm{Ge}_{0.86} \mathrm{Mn}_{0.1} \mathrm{Sn}_{0.04} \mathrm{Te}$, with parameters given in Table I. 


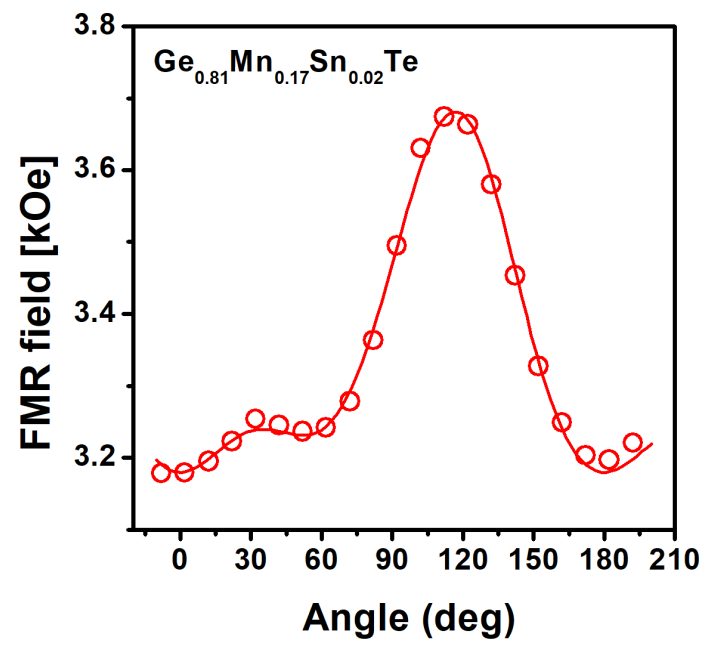

Fig. 4. Angular dependence of FMR peak positions in the (110) plane for the $\mathrm{Ge}_{0.81} \mathrm{Mn}_{0.17} \mathrm{Sn}_{0.02}$ Te layer at $12 \mathrm{~K}$. The solid line was calculated with parameters given in Table I.

splitting factor, and $F_{\theta \theta}, F_{\phi \phi}, F_{\theta \phi}$ are double derivates of the magnetic free-energy density $F$ after the polar, $\theta$, and azimuthal, $\phi$, angles of the magnetization vector $M$ relative to the crystal axes. Here $\theta_{e q}, \phi_{e q}$ are the equilibrium angles of the magnetization determined by minimizing $F$. The anisotropic part of $F$ in an applied magnetic field consists of the Zeeman energy $-\boldsymbol{H} \cdot \boldsymbol{M}$, the thin layer shape anisotropy $2 \pi M^{2} \cos ^{2} \theta$, and the trigonal pointsymmetry magnetocrystalline anisotropy energy (MAE), appropriate for rhombohedral lattices. For clarity, the contributions of cubic symmetry (with energy density parameter $\left.K_{4}\right)$ and of uniaxial symmetry $\left(K_{2}\right)$ are separated according to

$$
\begin{gathered}
F=-\boldsymbol{H} \cdot \boldsymbol{M}+2 \pi M^{2} \cos ^{2} \theta+K_{2} \sin ^{2} \theta \\
-\frac{2}{3} K_{4}\left(\sin ^{2} \theta-\frac{7}{8} \sin ^{4} \theta-\frac{\sqrt{2}}{2} \sin ^{3} \theta \cos \theta \sin (3 \phi)\right) .
\end{gathered}
$$

The fitting parameters are summarized in Table I.

TABLE I

Magnetization $M$ and anisotropy fields $K_{i} / M$ obtained from modeling the FMR data of Figs. 2 and 3. The resulting calculated angular dependence of the FMR fields using Eqs. (1) and (2) are depicted as solid lines in Figs. 2, 3.

\begin{tabular}{c|c|c|c|c}
\hline \hline$x_{M n}$ & $y_{S n}$ & $M\left[\mathrm{emu} / \mathrm{cm}^{3}\right]$ & $K_{2} / M[\mathrm{Oe}]$ & $K_{4} / M[\mathrm{Oe}]$ \\
\hline 0.15 & 0 & 36 & 823 & 0 \\
0.10 & 0.02 & 49 & 339 & -182 \\
0.17 & 0.04 & 50 & 337 & -144
\end{tabular}

The magnetocrystalline anisotropy in $\mathrm{Ge}_{1-x-y} \mathrm{Mn}_{x} \mathrm{Sn}_{y} \mathrm{Te}$ is very different from that in Sn free $\mathrm{Ge}_{1-x} \mathrm{Mn}_{x}$ Te layers with similar Mn content, as illustrated in Fig. 3. Already addition of $2 \%$ tin changes the anisotropy from purely uniaxial (in $\mathrm{Ge}_{0.85} \mathrm{Mn}_{0.15} \mathrm{Te}$ ) to distorted cubic (in $\mathrm{Ge}_{0.88} \mathrm{Mn}_{0.1} \mathrm{Sn}_{0.02} \mathrm{Te}$ ). A measurable contribution of cubic anisotropy was previously found in $\mathrm{Ge}_{1-x} \mathrm{Mn}_{x}$ Te layers with $x \geq 0.2$, which allowed distinction of two FE domains with oblique crystallographic axes rotated by $180^{\circ}$ around the [111] axis normal to the surface [8]. The FMR signal intensities of both domains were reversed when changing the orientation of the applied magnetic field. In $\mathrm{Ge}_{1-x-y} \mathrm{Mn}_{x} \mathrm{Sn}_{y} \mathrm{Te}$, however, only one FE domain is observed independent of the magnetic field direction. This suggests that addition of tin makes the crystal lattice more rigid suppressing magneto-elastic effects leading to domain reorientation.

\section{Summary}

We used the ferromagnetic resonance technique to study the impact of Sn codoping on the ferromagnetic and ferroelectric properties of the GeMnTe system. We found considerable changes in magnetocrystalline anisotropy as well as magneto-elastic effects.

\section{Acknowledgments}

Work supported by National Science Centre grant UMO-2014/15/B/ST3/03928.

\section{References}

[1] W. Eerenstein, N.D. Mathur, J.F. Scott, Nature 442, 759 (2006).

[2] S.-W. Cheong, M. Mostovoy, Nat. Mater. 6, 13 (2007).

[3] R. Ramesh, N.A. Spaldin, Nat. Mater. 6, 21 (2007).

[4] M. Gajek, M. Bibes, S. Fusil, K. Bouzehouane, J. Fontcuberta, A. Barthélémy, A. Fert, Nat. Mater. 6, 296 (2007).

[5] D.S. Jeong, R. Thomas, R.S. Katiyar, J.F. Scott, H. Kohlstedt, A. Petraru, C.S. Hwang, Rep. Prog. Phys. 75, 76502 (2012).

[6] N. Ortega, A. Kumar, J.F. Scott, R.S. Katiyar, J. Phys. C 27, 504002 (2015).

[7] N.A. Hill, J. Phys. Chem. B 104, 6694 (2000).

[8] R.W. Cochrane, M. Plischke, J.O. Ström-Olsen, Phys. Rev. B 9, 3013 (1974).

[9] H. Przybylińska, G. Springholz, R.T. Lechner, M. Hassan, M. Wegscheider, W. Jantsch, G. Bauer, Phys. Rev. Lett. 112, 047202 (2014).

[10] P.B. Littlewood, J. Phys. C Solid State Phys. 13 4875 (1980).

[11] A.J. Nadolny, J. Sadowski, T. Story, W. Dobrowolski, M. Arciszewska, K. Świątek, J. Kachniarz, J. Adamczewska, Acta Phys. Pol. A 94, 449 (1998).

[12] Ł. Kilański, M. Arciszewska, W. Dobrowolski, V. Domukhovski, V.E. Slynko, E.I. Slynko, J. Appl. Phys. 105, 103901 (2009). 